\title{
A Genetic Algorithm Approach to Solve Unit Commitment Problem
}

\author{
Aditya parashar ${ }^{1}$, Kuldeep Kumar Swankar ${ }^{2}$ \\ ${ }^{1}$ (Electrical Engg. Department, MITS Gwalior (M.P.), INDIA) \\ 2(Electrical Engg. Department, MITS Gwalior (M.P.), INDIA)
}

\begin{abstract}
In this a genetic algorithm based approach to resolve the thermal unit commitment (UC). During this approach load demand, the most objective of getting a possible unit combination for every loading condition whereas subjected to a range of constraints. The model during this study contains four-generation units and also the 8-hour daily load demand. The results are compared between the dynamic programming (DP) and genetic algorithm the achieved results prove the effectiveness, and validity of the planned approach to unravel the large-scale UC. In the results indicating comparison of the cost solutions is using the genetic algorithm and the Dynamic Programming. The MATLAB Tool box is used to find the result.
\end{abstract}

Keywords: Genetic Algorithm, Unit Commitment, Mat lab toolbox.

\section{Introduction}

It is the most important function of energy control center which determine the on/off status as well as the real power output of the generato $\$ \mathrm{hr}$ while minimizing the system operating cost over the planning period subject to various physical operation and computation constraints [1]. The Unit Commitment problem is a complex decision making process and it is difficult to develop any accurate mathematical optimization methods capable of solving the entire for any real size system. Also, multiple constraints should be imposed which must not be violated while finding the optimal or near optimal commitment schedule. Unit commitment (UC) is a nonlinear mixed integer optimization problem[2]. Genetic Algorithm (GA) is a general purpose stochastic and parallel search method, which can be used as an optimization technique for obtaining near-global optimum solutions of given problem. This algorithm is inspired from genetics and evolution theories of natural selection and survival of the fittest. It is an iterative procedure acting on a population of chromosomes, each chromosome being the encoding of a candidate solution to the problem. A fitness which depends on how well it solves the problem is associated with each chromosome [3]. Many pape $\$$ hr have given complete solution methods for unit commitment problem. These methods as Wood and Wollenberg, 1984.the famous traditional techniques such as Integer Programming (Michaelwich, 1990), Dynamic Programming (Tong et. al., 1991), Branch and Bound (wood and Wollenberg, 1984), Linear Programming (Tong et. al., 1991), Network flow programming (Tong et. al., 1991),Lagrangian relaxation (cheng et. at. 2000), and simulated annealing (Mantawy at. al. 1999). All these methods only provide near optimal solution. This solution affected by the limitation of the time and feasibility of the final solution[4]. Bakirtzis et al. (1996) have developed a genetic algorithm that uses different quality function technique to solve the unit commitment problem. The test result up to 100 generator units have been compared with that of Dynamic programming[5] and Lagrangian relaxation methods. Swarup (2002) have employed a new solution methodology to solve the Unit commitment problem using Genetic algorithm [6].

\section{Problem Promulation}

As is true for many systems, an electric power system experiences cycles. The demand for electricity is higher during the daytime and lower during the late evening and early morning. This cyclical demand requires that utilities companies plan for generation of power on hourly basis. The problem is fi $\$ /$ hrt to decide which of the available units to turn on, and then to determine an Economical dispatch schedule of the units. Determining an optimal Economical dispatch schedule of a set of generating Unit Commitment Problem (UCP).

\subsubsection{Fuel cost}

For a given system of $\mathrm{N}$ generation units at hour t, the total operating fuel cost, at that particular hour is minimized by economically dispatching the units subject to the following constraints:

(i) The total generated power by units equal to the used by load.

(ii) The power produced by each unit within bounded limits (minimum and maximum capacity).

This problem is called Economic Dispatch (ECD) and it can be stated as follows (the subscript $t$ is omitted for simplicity)

The $\mathrm{FC}_{\mathrm{i}}$ is the fuel cost function of the generator power output at any time interval.

$$
\mathrm{FC}_{\mathrm{i}}=\mathrm{a}_{\mathrm{i}}+\mathrm{b}_{\mathrm{i}} \mathrm{p}_{\mathrm{i}}+\mathrm{c}_{\mathrm{i}} \mathrm{p}_{\mathrm{i}}^{2}
$$


Where

$a_{i}, b_{i}, c_{i}$ represent unit cost coefficient.

$P_{i}$ is the $i$ unit power output [7]

$2, .1 .2$ starts- up cost

The start-up costs relate to a on units. If the thermal unit has been off for a long period, a cold start-up cost will be incurred. If the unit has been neoteric turned off (temperature of boiler is still high), a hot start-up cost is used.

The Generator start up cost depends on the time the unit has been off prior to start up. The startup cost in any given time interval can be represented by an exponential cost curve.

Where

$$
\mathrm{SC}_{\mathrm{i}}=\sigma_{\mathrm{i}}+\delta_{\mathrm{i}}\left\{1-\exp \left(-\mathrm{T}_{\mathrm{off}, i} / \tau_{\mathrm{i}}\right)\right\}
$$

$\sigma_{\mathrm{i}}$ is the hot start up cost.

$\delta$ I is the cold start up cost.

$\tau_{\mathrm{i}}$ is the unit cooling time constant.

$\mathrm{T}_{\mathrm{Offfi}}$ is the time a unit has been off.

The shut down cost, $\mathrm{SD}$ is usually given a constant value for each unit. The total cost of production, $\mathrm{F}_{\mathrm{T}}$ for the scheduling period is the sum of the all cost for all the units.

$$
\mathrm{F}_{\mathrm{T}, \mathrm{i}}=\sum_{t=1}^{T} \sum_{i=1}^{N} \quad \mathrm{FC}_{\mathrm{i}, \mathrm{t}}+\mathrm{SC}_{\mathrm{i}, \mathrm{t}}+\mathrm{SD}_{\mathrm{i}, \mathrm{t}}
$$

There are following constraints in unit commitment problem

(i) Power equality constraint, where the total power generated must be equal to power demand $\mathrm{P}_{\mathrm{D}}$ and system losses $\mathrm{P}_{\mathrm{L}}$. $\quad \sum_{i=0}^{N} \mathrm{P}_{\mathrm{i}} \mathrm{u}_{\mathrm{I}}-\left(\mathrm{P}_{\mathrm{D}}+\mathrm{P}_{\mathrm{L}}\right)=0 \quad \mathrm{t}=1,2, . . \mathrm{T}$

(ii) Hourly spinning reserve $\mathrm{R}$

$$
\sum_{i=0}^{N} \mathrm{P}_{\mathrm{i}}^{\max } \mathrm{u}_{\mathrm{i}^{-}}\left(\mathrm{P}_{\mathrm{D}}+\mathrm{P}_{\mathrm{L}}\right)=\mathrm{R} \quad \mathrm{t}=1,2, . . \mathrm{T}
$$

(iii) Generation limit constraint

$$
\mathrm{P}_{\mathrm{i}, \mathrm{t}}^{\min } \leq \mathrm{P}_{\mathrm{i}, \mathrm{t}} \leq \mathrm{P}_{\mathrm{i}, \mathrm{t}}^{\max }
$$

(iv) Start up cost

$$
\begin{gathered}
\left(T^{\text {on }-1, i}-M U T_{i}\right)\left(u_{t-1, i}-u_{t, i}\right) \geq 0 \\
\left(T^{\text {off }}{ }_{t-1, i}-M^{\prime} T_{i}\right)\left(u_{t, i}-u_{t-1, i}\right) \geq 0
\end{gathered}
$$

$\mathrm{T}^{\mathrm{on}}$ and $\mathrm{T}^{\text {off }}$ is the unit on and off time, while $\mathrm{u}_{\mathrm{t}, \mathrm{i}}$ is the unit on/ off $(1,0)$ state [4]

\section{Thermal Unit Commitment problem using Genetic algorithm}

For an application of Genetic Algorithm to the Unit commitment problem, a simple binary alphabet was chosen to encode a solution. If $\mathrm{N}$ represents the number of units and $\mathrm{H}$ the scheduling period in hou $\$ / \mathrm{hr}$, then with the assumption that at every hour a certain unit can be either ON or OFF, an $\mathrm{H}$ bit string is needed to describe the operation schedule of a single unit. In such a string, a ' 1 ' at a certain location indicates that the unit is $\mathrm{ON}$ at this particular hour while a ' 0 ' indicates that the unit is OFF. By concatenating the strings of $\mathrm{N}$ units, a $\mathrm{NH}$ bit string is formed. Then each genotype is evaluated, and its fitness value calculated as follows:

(i) The geno-type is decoded to an $\mathrm{N}$-unit / H-period operating schedule.

(ii) The fitness value $\mathrm{F}$, assigned to the geno type is given in (4.1)

$$
\mathrm{F}_{\mathrm{T}, \mathrm{i}}=\sum_{t=1}^{T} \sum_{i=1}^{N} \mathrm{FC}_{\mathrm{i}, \mathrm{t}}+\mathrm{SC}_{\mathrm{i}, \mathrm{t}}+\mathrm{SD}_{\mathrm{i}, \mathrm{t}}+\mathrm{PF}_{\mathrm{j}}
$$

$\mathrm{PF}_{\mathrm{j}}$ : penalty associated with violated constraint $\mathrm{j}$

(iii)The total fuel cost, $\mathrm{F}_{\mathrm{T}, \mathrm{i}}$ is computed as the sum of thehourly fuel costs by economically dispatching the load demand to the operating unit for every hour $t$, of the scheduling period. If for some hour $t$, due to the operating limits of the on line a unit, the power balance equation is not satisfied, a penalty term proportional to the power balance violation is added to the fitness function.

(iv)Time dependent startup costs and shutdown costs can be easily computed from the information contained in the genotype and the initial state continuous up or down time of units.

(v) For every violated constraint, a penalty term proportional to the amount of constraint violation is added to the fitness function. The penalty multiplie $\$ / h r, \mathrm{PF}_{\mathrm{j}}$ are chosen sufficiently large to discourage the selection of solution with violated constraints.

(vi)The great modeling flexibility of the GA solution to the unit commitment problem should be addressed at this point. In the GA solution, the unit commitment problem is not decomposed either by time or by unit [9].

\section{Result And Discussion}


The values of the daily load demand of the Four-generation-units of thermal power plant are adopted form ref. [10].

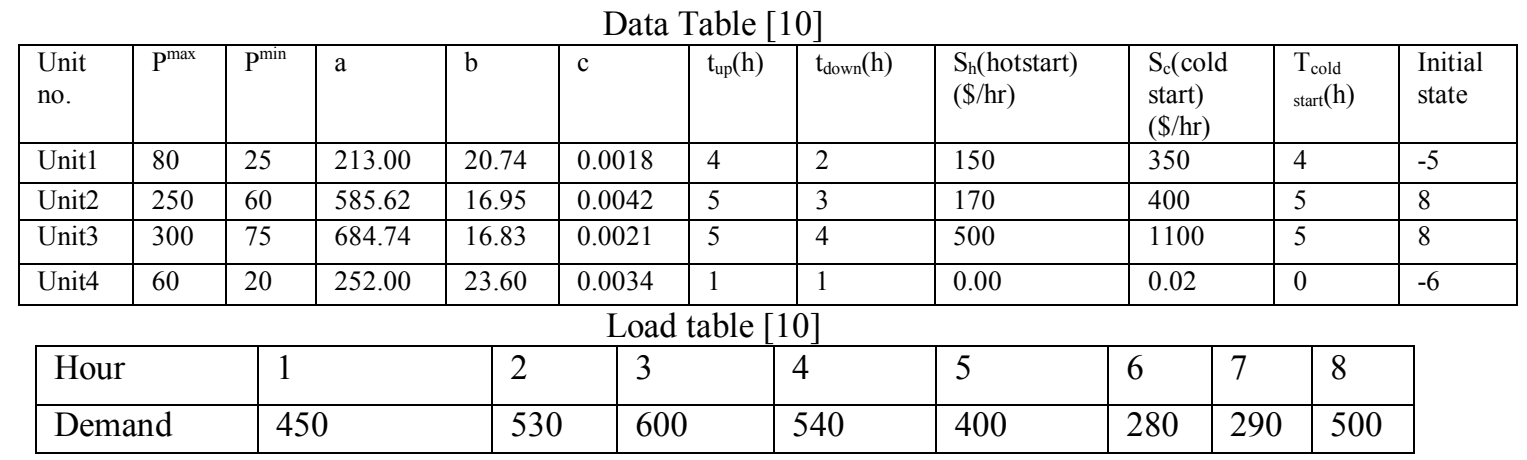

Simulation of Result

Hour 1, Load 450 MW

Dynamic programming $10579.89 \$ / \mathrm{hr}$

Genetic algorithm 10534.41 \$/hr

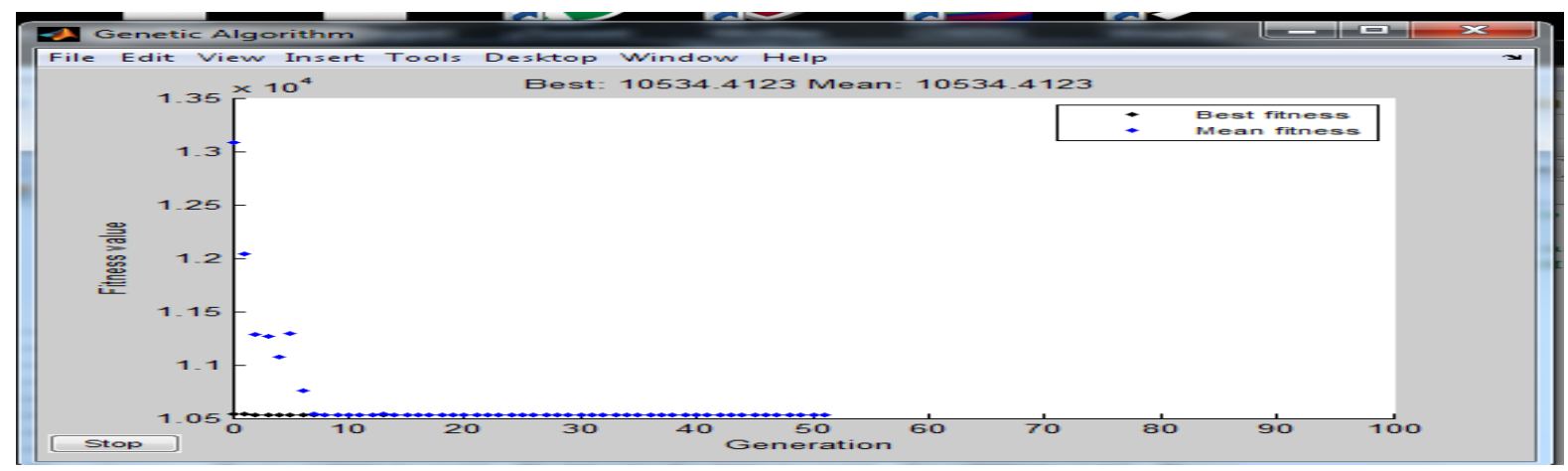

Fig.1 hour 1

\section{Hour 2, Load 530 MW}

Dynamic programming $11247.711 \$ / \mathrm{hr}$

Genetic algorithm 11222.44 \$/hr

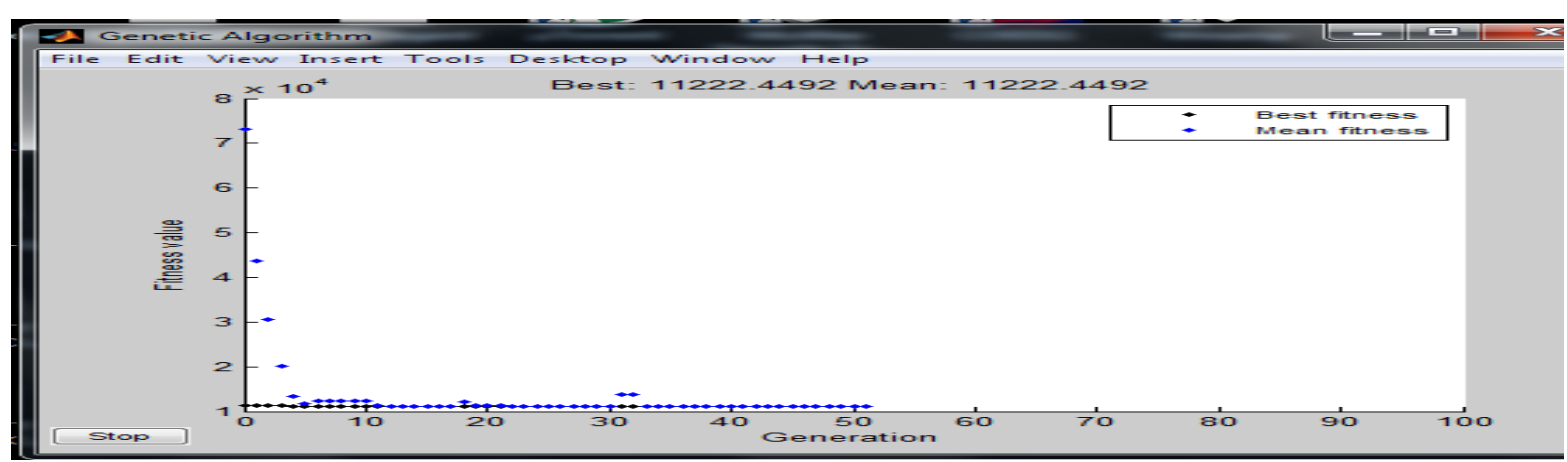

Fig. 2 hour 2

Hour 3, Load 600 MW

Dynamic programming $11460.08 \$ / \mathrm{hr}$

Genetic algorithm 11407.40 $\$ / \mathrm{hr}$ 


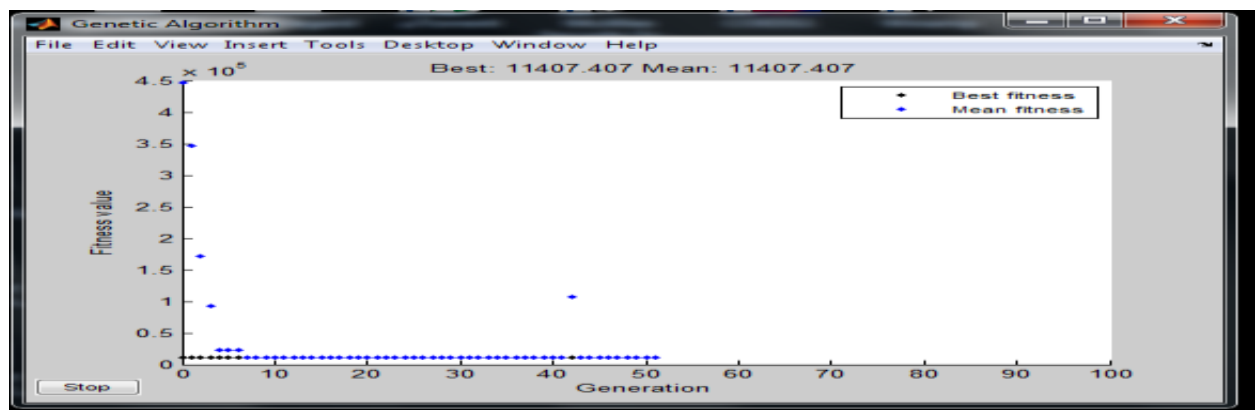

Fig.3 hour3

\section{Hour 4, Load 540 MW}

Dynamic programming $11153.68 \$ / \mathrm{hr}$

Genetic algorithm 11129.30 $\$ / \mathrm{hr}$

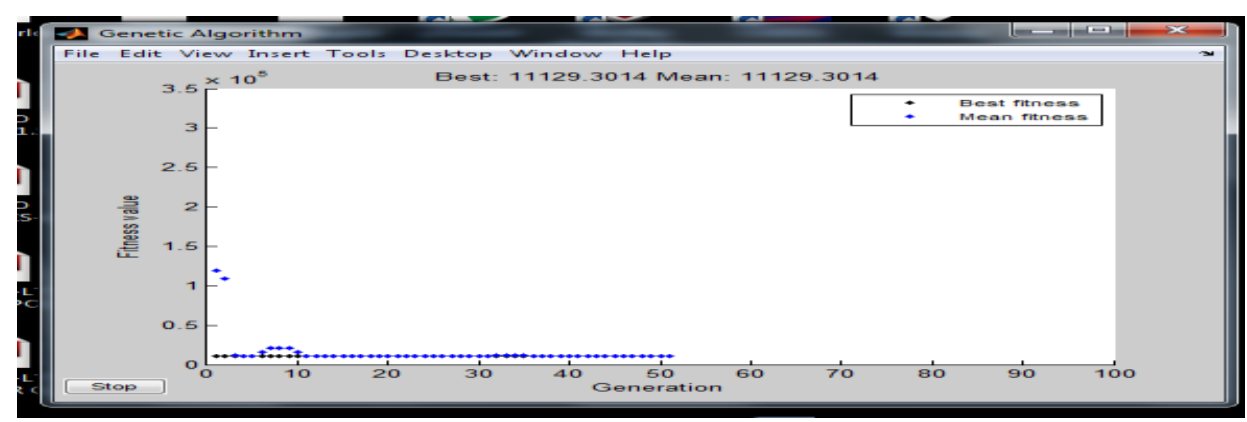

Fig.4 hour4

Hour 5, Load 400 MW

Dynamic programming $\mathbf{7 2 4 8 . 4 2} \$ / \mathrm{hr}$

Genetic algorithm 7231.70 $\$ / \mathrm{hr}$

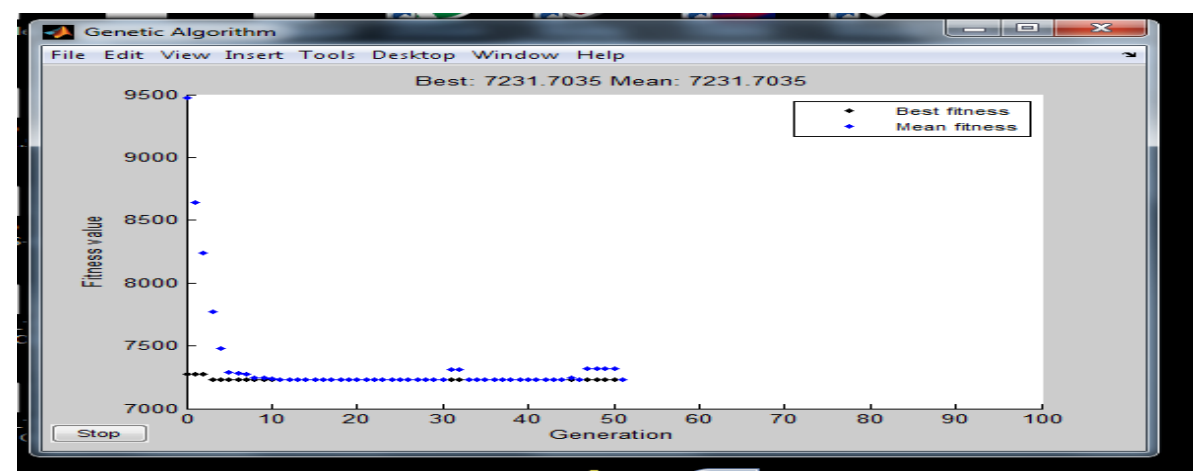

Fig.5 hour5

\section{Hour 6, Load 280 MW}

Dynamic programming 6036.03 $\$ / \mathrm{hr}$

Genetic algorithm 6013.67 \$/hr

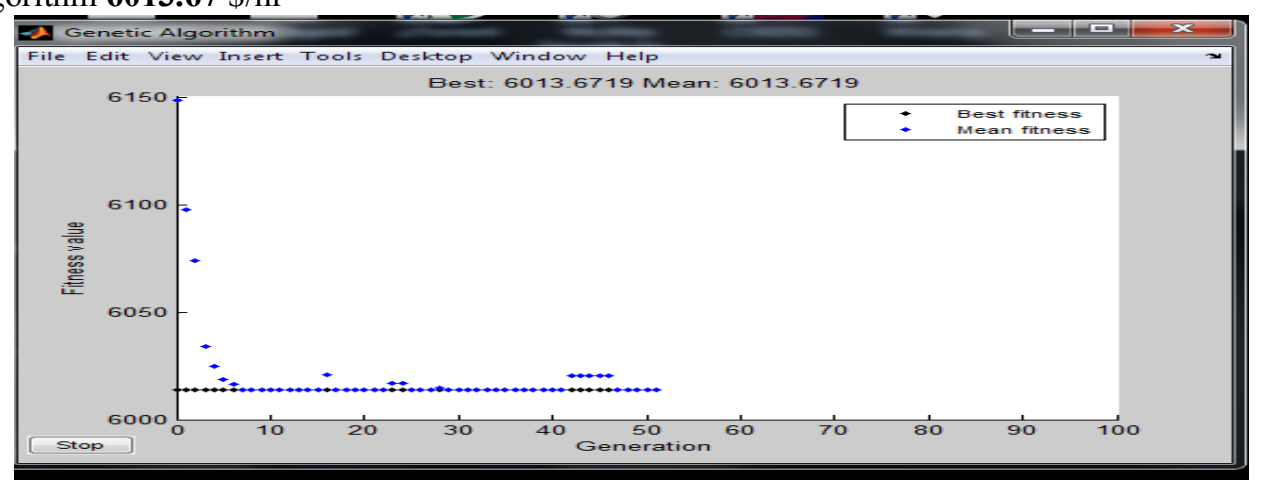

Fig.6 hour 6

Hour 7, Load 290 MW 
Dynamic programming 6207.00 $\$ / \mathrm{hr}$

Genetic algorithm 6179.73 $\$ / \mathrm{hr}$

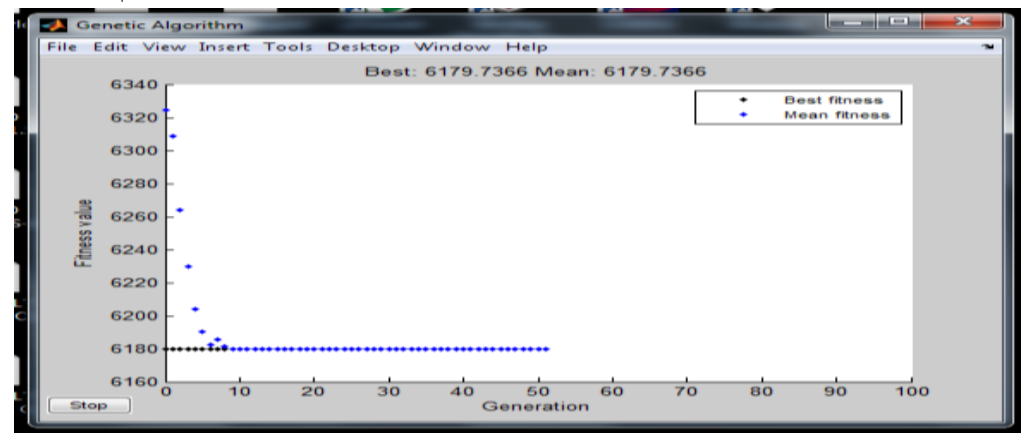

Hour 8, Load 500 MW

Fig.7 hour7

Dynamic programming 10659.78 $\$ / \mathrm{hr}$

Genetic algorithm 10617.89 \$/hr

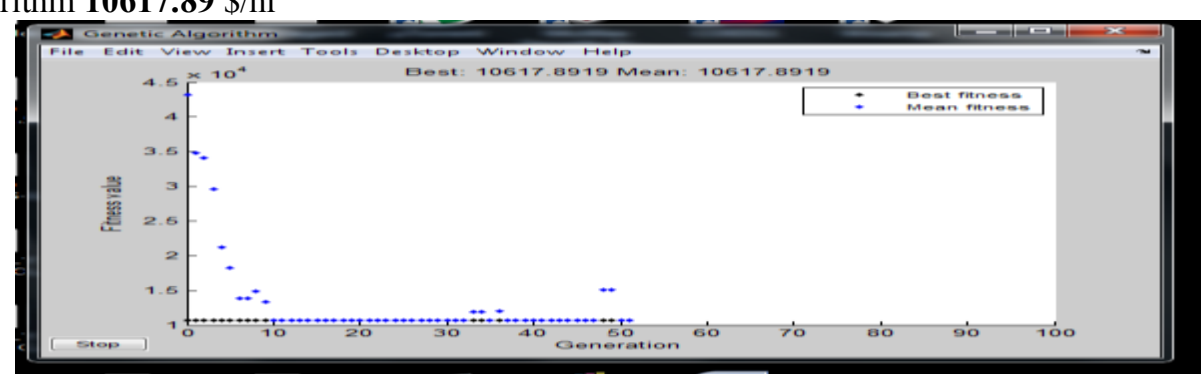

Fig. 8 hour 8

Final cost table

\begin{tabular}{|l|l|l|}
\hline Dynamic programming (\$/hr) & Genetic Algorithm $(\$ / \mathrm{hr})$ & Difference $(\$ / \mathrm{hr})$ \\
\hline 74591.81 & 74336.54 & 255.27 \\
\hline
\end{tabular}

\section{Conclusion}

The unit commitment problem can be solved by many methods to get optimal solution. The unit commitment problem is nothing; it is the simple study of load required at different time schedule. In this paper genetic algorithm can study and generated a better result to the dynamic programming method. A genetic algorithm can be used to solve unit commitment problem and reduced the total production cost of power generation.

\section{Journal Pape\$/hr:}

\section{References}

[1]. R. K. Santhi, S. Subramanian, "Adaptive Binary PSO based Unit Commitment",International Journal of Computer Applications (0975 - 8887)Volume 15- No.4, February 2011.

[2]. C. Christopher Columbus, Sishaj P. "Simon, Hybrid Particle swarm approach for Securityconstrained unit commitment",International Conference on Computing, Electronics and Electrical Technologies [ICCEET],2012.

[3]. T.Logenthiran,DiptiSrinivasan,"LRGAfo\$/hrolving Profit based GenerationScheduling Problem in Competitive Environment", 978$1-4244-7835-4 / 11 / \$ 26.00$ (2011 IEEE.

[4]. M.Sudhakaran,P.Ajay-D-Vimal Raj 'Integrating genetic algorithms and tabu search for unit commitment problem ',IJEST Vol.2,no.1,2010,pp.57-69.

[5]. S. A. Kazarlis, A. G. Bakirtzis, and V. Petridis, "A genetic algorithmsolution to the unit commitment problem," IEEE Transaction onPower System, vol. 11, pp. 83-92, Feburary 1996.

[6]. K. S. Swarup and S. Yamashiro," Unit Commitment Solution MethodologyUsing Genetic Algorithm", IEEE TRANSACTIONS ON POWER SYSTEMS, VOL. 17, NO. 1, FEBRUARY 2002

[7]. JorgeValenzuela \&AliceE.Smith 'A seeded mimetic for larg unit commitment problem'.

[8]. C.Christober Asir Rajan, M.R.Mohan,K.Manivannan ," Improved GeneticAlgorithm Solution to UnitCommitment Problem",0-78037525-4/02/\$17.00 02002 IEEE.

\section{Books:}

[10] A.J. Wood and B. Wollenberg, "Power Generation, Operation and Control", , 2 2n edition, John Wiley, New York, 1996.

\section{Journal Pape\$/hr:}

[11] Structured Genetic Algorithm Technique for Unit Commitment Problem by Kaveh Abookazemi*, Mohd Wazir Mustafa, Hussein Ahmad International Journal of Recent Trends in Engineering, Vol 1, No. 3, May 2009

[12] Rahul Garg, A.K.Sharma "Economic Generation and Scheduling of Power by Genetic Algorithm" Journal of Theoretical and Applied Information Technology, Vol. 4, No.12, pp-1142-1149, December-2008 